\title{
ELECTRIC RADIATION FROM CONDUCTORS
}

By H. M. MacDonald.

[Communicated January 14th, 1904. - Received February 23rd, 1904.]

THE rate at which energy is transferred across a closed surface into the space inside it is

$$
\begin{aligned}
\frac{1}{8 \pi} \frac{d}{d t} \iint\{F(m \gamma-n \beta) & +G(n \alpha-l \gamma)+H(l \beta-m \alpha)\} d S \\
& +\frac{1}{4 \pi} \iint\{X(m \gamma-n \beta)+Y(n \alpha-l \gamma)+Z(l \beta-m \alpha)\} d S, *
\end{aligned}
$$

where $\alpha, \beta, \gamma, X, Y, Z, F, G, H$ are the components of the magnetic force, the electric force, and the electrokinetic momentum at a point on the surface, and $l, m, n$ are the direction cosines of the normal to the surface at the point drawn outwards. The above expression does not give information as to the actual flow of energy across an element of the surface, but the flow across an element of the surface may be taken to be the corresponding element of the integral provided the results are applied to closed surfaces only. If, for simplicity, waves of one definite period are considered, it follows at once that there is no flow of energy across an element of surface which is perpendicular either to the electric force or to the magnetic force, the direction of the flow being perpendicular to both.

Now, at the surface of a perfect conductor the electric force at a point on the surface is either normal to the surface or vanishes; hence, at any instant of time the direction of the flow of energy just outside a perfect conductor is parallel to the surface of the conductor, except at points on it where the electric force vanishes. The electric force at the surface of the conductor will at any time vanish either along a definite number of closed lines on the surface or all over the surface.

The case in which the electric force vanishes along a number of closed lines and these lines are not fixed on the surface is that where there are sources outside the conductor; and the flow of the energy, except in the neighbourhood of these lines, is parallel to the surface of the conductor.

The case in which the electric force at the surface of the conductor 
always vanishes along a number of fixed lines on the surface is that where the electrical distribution on the surface is in a state of stationary oscillation, and in this case the electric force will vanish all over the surface once in each half period. At a time when the electric force vanishes all over the surface of the conductor there are surfaces outside it which, at previous times which are multiples of half a period, coincided with the surface of the conductor. If any one of these surfaces be followed as it advances from the conductor, it will trace out a family of surfaces, and the sufficient condition that there should be no radiation from the conductor is that the electric force tangential to each of these surfaces so traced out should vanish; for then there will be no transfer of energy across them.

Choosing then curvilinear coordinates $\xi, \eta, \xi$, where the surfaces $\hat{\xi}$ are the above surfaces, and $\eta, \xi$ define surfaces orthogonal to the surfaces $\xi$ and to each other, if $X, Y, Z, a, \beta, \gamma$ denote the components of the electric and magnetic forces normal to the surfaces $\xi, \eta, \xi$ at any point, and $V$ is the velocity of radiation, the equations satisfied by them are

$$
\begin{array}{ll}
\frac{\dot{X}}{V^{2}}=h_{2} h_{3}\left\{\frac{\partial}{\partial \eta}\left(\frac{\gamma}{h_{3}}\right)-\frac{\partial}{\partial \xi}\left(\frac{\beta}{h_{2}}\right)\right\}, & \dot{\alpha}=h_{2} h_{3}\left\{\frac{\partial}{\partial \xi}\left(\frac{Y}{h_{2}}\right)-\frac{\partial}{\partial \eta}\left(\frac{Z}{h_{3}}\right)\right\}, \\
\frac{\dot{Y}}{V^{2}}=h_{3} h_{1}\left\{\frac{\partial}{\partial \xi}\left(\frac{\alpha}{h_{1}}\right)-\frac{\partial}{\partial \xi}\left(\frac{\gamma}{h_{3}}\right)\right\}, & \dot{\beta}=h_{3} h_{1}\left\{\frac{\partial}{\partial \xi}\left(\frac{Z}{h_{3}}\right)-\frac{\partial}{\partial \xi}\left(\frac{X}{h_{1}}\right)\right\}, \\
\frac{\dot{Z}}{V^{2}}=h_{1} h_{2}\left\{\frac{\partial}{\partial \xi}\left(\frac{\beta}{h_{2}}\right)-\frac{\partial}{\partial \eta}\left(\frac{\alpha}{h_{1}}\right)\right\}, & \dot{\gamma}=h_{1} h_{2}\left\{\frac{\partial}{\partial \eta}\left(\frac{X}{h_{1}}\right)-\frac{\partial}{\partial \xi}\left(\frac{Y}{h_{2}}\right)\right\},
\end{array}
$$

where $d \xi / h_{1}, d \eta / h_{2}, d \xi / h_{3}$ are the elements of length normal to the surfaces $\xi, \eta, \xi$.

The above condition for no radiation gives $Y=0, Z=0$ : hence $a=0 ; \beta / h_{2}, \gamma / h_{3}$ are independent of $\hat{\xi}$; and $X$ satisfies the equation

$$
\frac{\ddot{X}}{V^{2}}=h_{2} h_{3}\left\{\frac{\partial}{\partial \eta} \frac{h_{1} h_{2}}{h_{3}} \frac{\partial}{\partial \eta}\left(\frac{X}{h_{1}}\right)+\frac{\partial}{\partial \xi} \frac{h_{3} h_{1}}{h_{2}} \frac{\partial}{\partial \xi}\left(\frac{X}{h_{1}}\right)\right\} ;
$$

whence $X / h_{2} h_{3}$ must be independent of $\xi$, and all possibilities are included in the following:- $h_{1} / h_{2}$ is independent of $\xi$ and $X / h_{1}$ is independent of $\eta$; the equation satisfied by $X$ being

$$
\frac{\ddot{X}}{V^{2}}=h_{2} h_{3} \frac{\partial}{\partial \xi} \frac{h_{3} h_{1}}{h_{2}} \frac{\partial}{\partial \xi}\left(\frac{X}{h_{1}}\right),
$$

and this requires that $h_{3}$ should be a function of $\xi$ only. Therefore the surfaces $\xi$ are either concentric spheres, concentric circular cylinders, 
or parallel planes. The last is the important case, and it appears that, if two parallel conducting planes be joined by any number of conducting cylinders whose generators are perpendicular to the planes, there are possible systems of electrical oscillations in the unlimited space which do not radiate away energy, a result which can be easily verified.*

From this it is to be expected that, when two nearly parallel conducting planes are connected by a conducting tubular surface which cuts the planes orthogonally, the generators being arcs of circles having their centres on the line of intersection of the planes, there will be possible systems of electrical oscillations in this space from which energy will be radiated away very slowly. It may be readily shown that, if $d$ be the mean length of the arcs of circles that bound the tube and $\rho$ their mean radius, the corrections to be added to the expressions for the electric and magnetic forces which would hold when the planes are parallel and the tube straight depend on powers of $d / \rho$, the lowest power being the second, and therefore, provided $d^{2} / \rho^{2}$ is negligible, the rate of decay of the oscillations can be neglected.

Another case in which it is to be expected that the rate of decay of the oscillations is very small is when the amount of bending is finite but the cross section of the tube is small; for the amount of radiation can only alter slowly as the cross section is diminished, provided the line integral of magnetic force looping the tube is kept constant, whereas the amount of energy in a finite space enclosing the tube increases rapidly.

The most important case is that of $a$ wire in the form of a closed circuit. The usual method of treatment is to evaluate the electric force tangential to the wire and in the direction of the wire, the part due to the wire being given as $B \log r_{0}$, where $r_{0}$ is the radius of the wire supposed to be of small circular cross section. $t$ It is then assumed that the condition for free oscillations is $B=0$. The expressions of the components of the electric force due to the wire are

$$
X=-\frac{\iota V}{4 \pi \kappa} \iint M\left[l_{1} \frac{\partial^{2}}{\partial x^{2}}+m_{1} \frac{\partial^{2}}{\partial x \partial y}+n_{1} \frac{\partial^{2}}{\partial x \partial z}+\kappa^{2} l_{1}\right] \frac{e^{-\iota \kappa l}}{R} d S, \quad \ldots,
$$

where $l_{1}, m_{1}, n_{1}$ are the direction cosines of the current at a point $x_{1}, y_{1}, z_{1}$ on the surface of the wire, $M$ is the magnetic force at the point, $R$ is the distance from the point $x_{1}, y_{1}, z_{1}$ to any point $x, y, z$, and the integrals are taken over the surface of the wire. Making the assumption, which is involved in the procedure above referred to, that $l_{1}, m_{1}, n_{1}$ are sensibly

* Cf. Lord Rayleigh, Phil. Mag., August, 1897.

†Cf. Poincaré, Les Oscillations électriques, pp. 233, 237. Paris, 1894. 
the same as the direction cosines of the circuit which is the limit of the wire when its cross section is indefinitely diminished, the expressions for the components of the electric force become

$$
X=-\frac{\iota V}{4 \pi \kappa} \int L\left(l_{1} \frac{\partial^{2}}{\partial x^{2}}+m_{1} \frac{\partial^{2}}{\partial x \partial y}+n_{1} \frac{\partial^{2}}{\partial x \partial z}+\kappa^{2} l_{1}\right) \frac{e^{\iota \kappa R}}{R} d s_{1}, \quad \ldots,
$$

where $L$ is the line integral of the magnetic force looping the wire and the integrals are now taken along the circuit. These expressions are equivalent to

$$
X=-\frac{\iota V}{4 \pi \kappa}\left\{\frac{\partial}{\partial x} \int \frac{e^{-\iota \kappa R}}{R} \frac{\partial I}{\partial s_{1}} d s_{1}+\kappa^{2} \int \frac{e^{-\imath \kappa R}}{R} l_{1} L d s_{1}\right\}, \quad \ldots,
$$

since $L$ is single-valued; whence the electric force tangential to the wire in the direction of the circuit is

$$
-\frac{\iota V}{4 \pi \kappa}\left\lceil\frac{\partial}{\partial s} \int \frac{e^{-\imath \kappa R}}{R} \frac{\partial L}{\partial s_{1}} d s_{1}+\kappa^{2} \int \frac{e^{-\imath \kappa R}}{R}\left(l l_{1}+m m_{1}+n n_{1}\right) L d s_{1},\right.
$$

where $l, m, n$ are the direction cosines of the circuit at the point corresponding to the point on the wire where the electric force is being evaluated and $\partial / \partial s$ denotes differentiation in this direction. The condition that this expression should not become infinite when $r_{0}$, the radius of the wire, is made to vanish is that $L$ should satisfy the equation

$$
\frac{\partial^{2} L}{\partial s^{2}}+\kappa^{2} L=0,
$$

which is the condition $B=0$ given above; but, this condition being satisfied, the value of the above expression is in general a finite quantity different from zero. Hence, a circuit of Hertzian oscillators is not in general the limit of a conducting wire. For example, in the case of a circuit in the form of a circle of radius $c$, if $\theta$ be the angle the radius to any point makes with a fixed radius, the condition that the electric force tangential to the circuit at a point on it is not infinite gives

$$
L=A \cos \kappa c \theta+B \sin \kappa c \theta,
$$

and, since $L$ is single-valued, $\kappa c$ must be an integer $n$. The value of the electric force tangential to the circuit at the point $\theta$ on it is then, from the previous expression,

$$
-\frac{\iota V}{4 \pi \kappa}\left\{\frac{1}{c} \frac{\partial}{\partial \theta} \int_{0}^{2 \pi} \frac{e^{-\imath \kappa R}}{R} \frac{\partial L}{\partial \theta_{1}} d \theta_{1}+\kappa^{2} c \int_{0}^{2 \pi} \frac{e^{-\iota \kappa R}}{R} L \cos \left(\theta-\theta_{1}\right) d \theta_{1}\right\},
$$

that is

$$
-\frac{\iota V}{4 \pi \kappa c} \int_{0}^{2 \pi}\left\{\frac{\partial^{2} L}{\partial \theta_{1}^{2}}+\kappa^{2} c^{2} L \cos \left(\theta-\theta_{1}\right)\right\} \frac{e^{-\iota \kappa R}}{R} d \theta,
$$


or, writing for $\kappa c$ its value $n$,

$$
\begin{aligned}
& \frac{\iota m V}{4 \pi c}\left[\int_{0}^{\theta}\left(A \cos n \theta_{1}+B \sin n \theta_{1}\right) \sin \frac{1}{2}\left(\theta-\theta_{1}\right) e^{-2 \imath n \sin \frac{1}{2}\left(\theta-\theta_{1}\right)} d \theta_{1}\right. \\
& \left.\quad+\int_{0}^{2 \pi}\left(A \cos n \theta_{1}+B \sin n \theta_{1}\right) \sin \frac{1}{2}\left(\theta_{1}-\theta\right) e^{-2 \imath \sin \frac{1}{2}\left(\theta_{1}-\theta\right)} d \theta_{1}\right],
\end{aligned}
$$

which is $\frac{\imath n V}{2 \pi c} \int_{0}^{\pi}\{A \cos n(\theta-2 \chi)+B \sin n(\theta-2 \chi)\} \sin \chi e^{-2 \iota n \sin x} d \chi$,

or

$$
\frac{n V}{4 c}\left\{J_{2 n+1}(2 n)-J_{2 n-1}(2 n)\right\}\{A \cos n \theta+B \sin n \theta\},
$$

where $J_{n}(x)$ denotes Bessel's function of order $n$; thus the electric force tangential to the circuit at a point on it is a finite quantity.

The radiation from a circuit of Hertzian oscillators arranged according to the law $d^{2} L / d s^{2}+\kappa^{2} L=0$ can be found as follows. Let a tubular surface be drawn enclosing the circuit: then, the radiation across all such tubular surfaces being the same, the rate of radiation of energy from the circuit is equal to the value of the integral

$$
\begin{aligned}
-\frac{1}{8 \pi} \frac{d}{d t} \iint\{F(m \gamma-n \beta)+G(n \alpha-l \gamma)+H(l \beta-m \alpha)\} d S \\
-\frac{1}{4 \pi} \iint\{X(m \gamma-n \beta)+Y(n \alpha-l \gamma)+Z(l \beta-m \alpha)\} d S
\end{aligned}
$$

taken over any one of these tubular surfaces, and is therefore equal to the limit that this integral attains when the cross section of the tube is indefinitely diminished; the value of this limit is $-\frac{1}{8 \pi} \int L_{1} E_{1} d s$ where $L_{1}$ is the maximum value of $L$ and $E_{1}$ the maximum value of the electric force tangential to the circuit at a point on it, the integral being taken once round the circuit. Hence the rate of radiation of energy from the circuit is $-\frac{1}{8 \pi} \int L_{1} E_{1} d s$.

When the circuit is a circle of radius $c$ this becomes, from a preceding result,

$$
\frac{n V}{32 \pi c}\left\{J_{2 n-1}(2 n)-J_{2 n+1}(2 n)\right\} \int_{0}^{2 \pi}\left\{A_{1} \cos n \theta+B_{1} \sin n \theta\right\}^{2} c d \theta_{1}
$$

where $A_{1}, B_{1}$ are the maximum values of $A$ and $B$, that is

$$
\frac{n V}{32}\left\{J_{2 n-1}(2 n)-J_{2 n+1}(2 n)\right\}\left(A_{1}^{2}+B_{1}^{2}\right) .
$$


This result shows that the rate of radiation from a circle of Hertzian oscillators of given maximum amplitude arranged according to the above law is, for oscillations having the same number of nodes, independent of the radius of the circuit. For the oscillations of greatest wave-length $n=1$, and the rate of radiation is

$$
\frac{V}{32}\left\{J_{1}(2)-J_{3}(2)\right\}\left(A_{1}^{2}+B_{1}^{2}\right),
$$

that is $014 V\left(A_{1}^{2}+B_{1}^{2}\right)$ approximately.

It has been seen above that a circuit of Hertzian oscillators is not the limit of a conducting wire; it also appears from the following considerations that a distribution of oscillators on the surface of the wire, each oscillator being parallel to the tangent to the circuit at the corresponding point on it, does not give an approximate solution. Taking the case of a wire in the form of a circle, the radius of the circle which is the central axis of the wire being $c$ and the radius of the cross section being $r_{0}$, the above distribution is equivalent to a bundle of circuits of Hertzian oscillators, each circuit being on the surface of the wire and parallel to the circuit forming its central axis. Let $P$ be a point on any one of these circuits, $Q$ a point near to $P$ but not in the plane of the circuit through $P$; then the electric force due to this circuit at $Q$ is made up of the following - a force along $P Q$ of the order $P Q^{-1}$, a force parallel to the tangent to the circuit at $P$ of the order $c^{-1}$, and a force perpendicular to the tangent and parallel to the plane of the circuit of the order $c^{-1}$. If $Q$ is on the surface of the wire, the distribution can be arranged so that the resultant of the first group is normal to the surface of the wire and of order $r_{0}^{-1}$, but there will remain two tangential forces each of order $c^{-1}$, one in the direction parallel to the tangent to the central axis of the wire at the corresponding point on it, the other perpendicular to this direction, the latter force only vanishing when $Q$ is in the plane of the central axis of the wire. The tangential force parallel to the central axis of the wire could be made to vanish by making the period complex, but the force tangential to the cross section which is of the same order would still remain. For the oscillations of greatest wave-length the argument can be put more simply as follows:- If $P$ is a point on the surface of the wire, $O$ the corresponding point on the central axis of the wire, the unbalanced tangential force at $P$ parallel to the tangent to the central axis at $O$ is largely contributed by the oscillators situated near $O_{1}$, the extremity of the diameter through $O$ of the circle that forms the central axis of the wire, and the unbalanced tangential force per- 
pendicular to the tangent at $O$ is largely contributed by the oscillators situated near $O_{2}$ and $O_{3}, O_{2} O_{3}$ being the diameter of the circle forming the central axis perpendicular to $O O_{1}$, the parts contributed by an oscillator near $\mathrm{O}_{2}$ and one similarly placed near $\mathrm{O}_{3}$ being additive, and the forces in both cases are of the same order.

A direct method of attempting to solve the problem of the oscillations of an electrical distribution on a wire in the form of a circle is the following. Let cylindrical coordinates $r, \theta, z$ be chosen, the axis of $z$ being the axis of the circle that forms the central axis of the wire, and let the transformation $r+\iota z=f(\xi+\imath \eta)$ be found such that one of the surfaces $\eta$ is the surface of the wire; then the equations satisfied by the components of the electric and magnetic forces are

$$
\begin{array}{ll}
\frac{\dot{X}}{\bar{V}^{2}}=\frac{J}{r}\left[\frac{\partial}{\partial \eta}(\gamma r)-\frac{\partial}{\partial \theta}\left(\frac{\beta}{J}\right)\right], & \dot{\alpha}=\frac{J}{r}\left[\frac{\partial}{\partial \theta}\left(\frac{Y}{J}\right)-\frac{\partial}{\partial \eta}(Z r)\right], \\
\frac{\dot{Y}}{V^{2}}=\frac{J}{r}\left[\frac{\partial}{\partial \theta}\left(\frac{\alpha}{J}\right)-\frac{\partial}{\partial \xi}(\gamma r)\right], & \dot{\beta}=\frac{J}{r}\left[\frac{\partial}{\partial \xi}(Z r)-\frac{\partial}{\partial \theta}\left(\frac{X}{J}\right)\right], \\
\frac{\dot{Z}}{\bar{V}^{2}}=J^{2}\left[\frac{\partial}{\partial \xi}\left(\frac{\beta}{J}\right)-\frac{\partial}{\partial \eta}\left(\frac{\alpha}{J}\right)\right], & \dot{\gamma}=J^{2}\left[\frac{\partial}{\partial \eta}\left(\frac{X}{J}\right)-\frac{\partial}{\partial \xi}\left(\frac{Y}{J}\right)\right],
\end{array}
$$

where

$$
J^{2}=\frac{\partial(\xi, \eta)}{\partial(r, z)} .
$$

For the oscillations of greatest wave-length

$$
\begin{array}{lll}
\alpha=\alpha_{1} \cos \theta, & \beta=\beta_{1} \cos \theta, & \gamma=\gamma_{1} \sin \theta, \\
X=X_{1} \sin \theta, & Y=Y_{1} \sin \theta, & Z=Z_{1} \cos \theta,
\end{array}
$$

and $X_{1}, Y_{1}, Z_{1}, a_{1}, \beta_{1}, \gamma_{1}$ satisfy the equations

$$
\begin{array}{rlr}
\frac{\dot{X}_{1}}{V^{2}}=\frac{J}{r}\left[\frac{\partial}{\partial \eta}\left(\gamma_{1} r\right)+\frac{B_{1}}{J}\right], & \dot{a}_{1}=\frac{J}{r}\left[\frac{Y_{1}}{J}-\frac{\partial}{\partial \eta}\left(Z_{1} r\right)\right], \\
\frac{\dot{Y}_{1}}{V^{2}}=-\frac{J}{r}\left[\frac{\partial}{\partial \xi}\left(\gamma_{1} r+\right) \frac{a_{1}}{J}\right], & \dot{\beta}_{1}=\frac{J}{r}\left[\frac{\partial}{\partial \xi}\left(Z_{1} r\right)-\frac{X_{1}}{J}\right], \\
\frac{\dot{Z}_{1}}{V^{2}}=J^{2}\left[\frac{\partial}{\partial \xi}\left(\frac{\beta_{1}}{J}\right)-\frac{\partial}{\partial \eta}\left(\frac{a_{1}}{J}\right)\right], & \dot{\gamma}_{1}=J^{2}\left[\frac{\partial}{\partial \eta}\left(\frac{X_{1}}{J}\right)-\frac{\partial}{\partial \xi}\left(\frac{Y_{1}}{J}\right)\right] ; \\
\text { whence } \quad X_{1}=J \frac{\partial}{\partial \xi}\left(Z_{1} r\right)-r \dot{\beta}_{1}, & Y_{1}=J \frac{\partial}{\partial \eta}\left(Z_{1} r\right)+r a_{1} ;
\end{array}
$$


therefore

$$
\begin{aligned}
& J \frac{\partial}{\partial \xi}\left(\frac{\dot{Z}_{1} r}{V^{2}}\right)-\frac{r \ddot{\beta}_{1}}{V^{2}}=\frac{J}{r}\left[\frac{\partial}{\partial \eta}\left(\gamma_{1} r\right)+\frac{\beta_{1}}{J}\right], \\
& J \frac{\partial}{\partial \eta}\left(\frac{\dot{Z}_{1} r}{V^{2}}\right)+\frac{r \alpha_{1}}{V^{2}}=-\frac{J}{r}\left[\frac{\partial}{\partial \xi}\left(\gamma_{1} r\right)+\frac{\alpha_{1}}{J}\right]
\end{aligned}
$$

that is

$$
\begin{aligned}
& \alpha_{1}\left(\kappa^{2} r^{2}-1\right)=J\left[r \frac{\partial}{\partial \eta}\left(\frac{\dot{Z}_{1} r}{V^{2}}\right)+\frac{\partial}{\partial \xi}\left(\gamma_{1} r\right)\right] \\
& \beta_{1}\left(\kappa^{2} r^{2}-1\right)=J\left[-r \cdot \frac{\partial}{\partial \xi}\left(\frac{\dot{Z}_{1} r}{V^{2}}\right)+\frac{\partial}{\partial \eta}\left(\gamma_{1} r\right)\right]
\end{aligned}
$$

hence

$$
\begin{aligned}
J^{2} \frac{\partial}{\partial \xi}\left\{\frac{r}{\kappa^{2} r^{2}-1} \frac{\partial}{\partial \xi}\left(\frac{\dot{Z}_{1} r}{V^{2}}\right)\right\} & +J^{2} \frac{\partial}{\partial \eta}\left\{\frac{r}{\kappa^{2} \gamma^{2}-1} \frac{\partial}{\partial \eta} \frac{\dot{Z}_{1} r}{V^{2}}\right\}+\frac{\dot{Z}_{1}}{V^{2}} \\
& =J^{2}\left[\frac{\partial}{\partial \eta}\left(\gamma_{1} r\right) \frac{\partial}{\partial \xi} \frac{1}{\kappa^{2} r^{2}-1}-\frac{\partial}{\partial \xi}\left(\gamma_{1} r\right) \frac{\partial}{\partial \eta} \frac{1}{\kappa^{2} r^{2}-1}\right] ;
\end{aligned}
$$

and, similarly,

$$
\begin{aligned}
J^{2} \frac{\partial}{\partial \xi}\left\{\frac{r}{\kappa^{2} r^{2}-1} \frac{\partial}{\partial \dot{\xi}}\left(\dot{\gamma}_{1} r\right)\right\} & +J^{2} \frac{\partial}{\partial \eta}\left\{\frac{r}{\kappa^{2} r^{2}-1} \frac{\partial}{\partial \eta}\left(\dot{\gamma}_{1} r\right)\right\}+\dot{\gamma}_{1} \\
& =J^{2}\left[\frac{\partial}{\partial \eta}\left(Z_{1} r\right) \frac{\partial}{\partial \xi} \frac{1}{\kappa^{2} r^{2}-1}-\frac{\partial}{\partial \xi}\left(Z_{1} r\right) \frac{\partial}{\partial \eta} \frac{1}{\kappa^{2} r^{2}-1}\right] .
\end{aligned}
$$

Now the transformation $r+\imath z=f(\xi+\iota \eta)$ conformably represents any one of the planes through the axis of the ring on a strip of the $\xi$, $\eta$ plane parallel to the axis of $\eta$; the breadth of this strip can be chosen to be $\pi$, and therefore it may be assumed that

$$
Z_{1}=\Sigma Z_{n}^{\prime} \cos n \xi, \quad \gamma_{1}=\Sigma \gamma_{n}^{\prime} \sin n \xi,
$$

where the values of $n$ are integers. When these expressions are substituted in the above two equations and in the boundary conditions $X=0, Z=0$ when $\eta=\eta_{0}$, the resulting equations aro only sufficient to determine $Z_{n}^{\prime}$ and $\gamma_{n}^{\prime}$, and it will remain to determine $\kappa^{2}$ from the condition that the expression for $Z$ or $\gamma$ converges for all values of $\eta$ lying between 0 and $\eta_{0}$. The resulting analysis would prove complicated, and it is doubtful whether any useful result could be obtained while sufficient information for practical purposes can be obtained otherwise.

Restricting the problem to the case where the cross section of the wire is circular and of small radius $r_{0}$, approximations for the electric and magnetic forces in the immediate neighbourhood of the wire can be found. For this purpose it is convenient to adopt a different system of coordinates; choosing as coordinates the shortest distance $r$ of a point from the central axis of the wire, the angle $\phi$ this shortest distance makes 
with the plane of the central axis, and the angle $\theta$ the plane through the axis of the ring and the shortest distance makes with a fixed plane through the axis of the ring, then $X, Y, Z, \alpha, \beta, \gamma$ being the components of the electric force and the magnetic force corresponding to the coordinates $r, \theta, \phi$, the equations satisfied by them are

$$
\begin{array}{lll}
\frac{\dot{X}}{V^{2}}=\frac{1}{\varpi r}\left[\frac{\partial}{\partial \theta}(\gamma r)-\frac{\partial}{\partial \phi}(\beta \varpi)\right], & \dot{\alpha}=\frac{1}{\varpi r}\left[\frac{\partial}{\partial \phi}(\gamma \varpi)-\frac{\partial}{\partial \theta}(Z r)\right], \\
\frac{\dot{Y}}{V^{2}}=\frac{1}{r}\left[\frac{\partial \alpha}{\partial \phi}-\frac{\partial}{\partial r}(\gamma r)\right], & \dot{\beta}=\frac{1}{r}\left[\frac{\partial}{\partial r}(Z r)-\frac{\partial \chi}{\partial \phi}\right], \\
\frac{\dot{Z}}{V^{2}}=\frac{1}{\varpi}\left[\frac{\partial}{\partial r}(\beta \varpi)-\frac{\partial \alpha}{\partial \theta}\right], & \dot{\gamma}=\frac{1}{\varpi}\left[\frac{\partial \chi}{\partial \theta}-\frac{\partial}{\partial r}(\gamma \varpi)\right],
\end{array}
$$

in which $\varpi$ has been written for $c+r \cos \phi$. For the oscillations of greatest wave-length

$$
\begin{array}{lll}
\alpha=\alpha^{\prime} \cos \theta, & \beta=\beta^{\prime} \sin \theta, & \gamma=\gamma^{\prime} \cos \theta, \\
X=X^{\prime} \sin \theta, & Y=Y^{\prime} \cos \theta, & Z=Z^{\prime} \sin \theta,
\end{array}
$$

whence the above equations become

$$
\begin{array}{ll}
\frac{\dot{X}^{\prime}}{V^{2}}=-\frac{1}{\varpi r}\left[\gamma^{\prime} r+\frac{\partial}{\partial \phi}\left(\beta^{\prime} \varpi\right)\right], & \dot{\alpha}^{\prime}=\frac{1}{\varpi r}\left[\frac{\partial}{\partial \phi}\left(Y^{\prime} \varpi\right)-Z^{\prime} r\right], \\
\frac{\dot{Y}^{\prime}}{V^{2}}=\frac{1}{r}\left[\frac{\partial \alpha^{\prime}}{\partial \phi}-\frac{\partial}{\partial r}\left(\gamma^{\prime} r^{\prime}\right)\right], & \dot{\beta}^{\prime}=\frac{1}{r}\left[\frac{\partial}{\partial r^{\prime}}\left(Z^{\prime} r\right)-\frac{\partial X^{\prime}}{\partial \phi}\right], \\
\frac{\dot{Z}^{\prime}}{V^{2}}=\frac{1}{\varpi}\left[\frac{\partial}{\partial r}\left(\beta^{\prime} \varpi\right)+\alpha^{\prime}\right], & \dot{\gamma}^{\prime}=\frac{1}{\varpi}\left[X^{\prime}-\frac{\partial}{\partial r}\left(Y^{\prime} \varpi\right)\right],
\end{array}
$$

and, eliminating $X^{\prime}, Y^{\prime}, Z^{\prime}$,

$$
\begin{aligned}
& -\kappa^{2} \alpha^{\prime}=\frac{1}{\varpi r}\left[\frac{\partial}{\partial \phi} \frac{\varpi}{r}\left\{\frac{\partial \alpha^{\prime}}{\partial \phi}-\frac{\partial}{\partial r}\left(\gamma^{\prime} r\right)\right\}-\frac{v}{\varpi}\left\{\frac{\partial}{\partial r}\left(\beta^{\prime} \varpi\right)+\alpha^{\prime}\right\}\right], \\
& -\kappa^{2} \beta^{\prime}=\frac{1}{r}\left[\frac{\partial}{\partial r} \frac{r}{\varpi}\left\{\frac{\partial}{\partial r}\left(\beta^{\prime} \varpi\right)+\alpha^{\prime}\right\}+\frac{\partial}{\partial \phi} \frac{1}{\varpi r}\left\{\gamma^{\prime} r+\frac{\partial}{\partial \phi}\left(\beta^{\prime} \varpi\right)\right\}\right], \\
& -\kappa^{2} \gamma^{\prime}=\frac{1}{\varpi}\left[-\frac{1}{\varpi r}\left\{\gamma^{\prime} r+\frac{\partial}{\partial \phi}\left(\beta^{\prime} \varpi\right)\right\}-\frac{\partial}{\partial r} \frac{\varpi}{r}\left\{\frac{\partial \alpha^{\prime}}{\partial \phi}-\frac{\partial}{\partial r}\left(\gamma^{\prime} r\right)\right\}\right],
\end{aligned}
$$

and any one of these three equations can be replaced by the equation

$$
\frac{\partial}{\partial r}\left(\alpha^{\prime} \varpi r\right)+\beta^{\prime} r+\frac{\partial}{\partial \phi}\left(\gamma^{\prime} \varpi\right)=0 .
$$


Taking the first and third of the above group, they can be written

$$
\begin{aligned}
& \left(1-\kappa^{2} \varpi^{2}\right) \alpha^{\prime}=\frac{\varpi}{r} \frac{\partial}{\partial \phi}\left(\frac{\varpi}{r} \frac{\partial \alpha^{\prime}}{\partial \phi}\right)-\frac{\varpi}{r} \frac{\partial}{\partial \phi}\left\{\frac{\varpi}{r} \frac{\partial}{\partial r}\left(\gamma^{\prime} r\right)\right\}-\frac{\partial}{\partial r}\left(\beta^{\prime} \varpi\right), \\
& \left(1-\kappa^{2} \varpi^{2}\right) \gamma^{\prime}=\varpi \frac{\partial}{\partial r}\left\{\frac{\varpi}{r} \frac{\partial}{\partial r}\left(\gamma^{\prime} r\right)\right\}-\varpi \frac{\partial}{\partial r}\left\{\frac{\varpi}{r} \frac{\partial \alpha^{\prime}}{\partial \phi}\right\}-\frac{1}{r} \frac{\partial}{\partial \phi}\left(\beta^{\prime} \varpi\right) .
\end{aligned}
$$

Writing

$\alpha^{\prime}=\sum_{1}^{\infty} \alpha_{n} \sin n \phi, \quad \beta^{\prime}=\sum_{1}^{\infty} \beta_{n} \sin n \phi, \quad \gamma^{\prime}=\gamma_{0}+\sum_{1}^{\infty} \gamma_{n} \cos n \phi$,

and assuming that when $r / c$ is small $a_{n+1}, \beta_{n+1}, \gamma_{n+1}$ are small compared with $\alpha_{n}, \beta_{n}, \gamma_{n}$, the values of $\alpha_{n}, \beta_{n}, \gamma_{n}$ can be successively calculated to any desired degree of approximation from the above equations together with the boundary conditions $Y^{\prime}=0, Z^{\prime}=0$ when $r=r_{0}$, that is $\alpha^{\prime}=0$, $\frac{\partial}{\partial r}\left(\beta^{\prime} \varpi\right)=0, \frac{\partial}{\partial r}\left(\gamma^{\prime} r\right)=0$, when $r=r_{0}$. The most important part of $\gamma_{0}$ is determined by the equation

$$
\left(1-\kappa^{2} c^{2}\right) \gamma_{0}=c^{2} \frac{\partial}{\partial r} \frac{1}{r} \frac{\partial}{\partial r}\left(r \gamma_{0}\right)
$$

subject to the condition that $\frac{\partial}{\partial r}\left(r \gamma_{0}\right)=0$, when $r=r_{0}$; whence the most important part of $\gamma_{0}$ is given by $\gamma_{0}=A / r$, and $1-\kappa^{2} c^{2}$ must be a small quantity. The most important parts of $a_{1}, \beta_{1}, \gamma_{1}$ are determined by the equations

$$
\begin{gathered}
\frac{\partial}{\partial r}\left(a_{1} c r\right)+\beta_{1} r-\gamma_{1} c-\gamma_{0} r=0 \\
-\frac{c^{2}}{r^{2}} a_{1}+\frac{c^{2}}{r^{2}} \frac{\partial}{\partial r}\left(\gamma_{1} r\right)+\frac{c}{r} \frac{\partial}{\partial r}\left(\gamma_{0} r\right)-c \frac{\partial \beta_{1}}{\partial r}=0
\end{gathered}
$$

$c^{2} \frac{\partial}{\partial r} \frac{1}{r} \frac{\partial}{\partial r}\left(\gamma_{1} r\right)+r c \frac{\partial}{\partial r} \frac{1}{r} \frac{\partial}{\partial r}\left(\gamma_{0} r\right)+c \frac{\partial^{2}}{\partial r^{2}}\left(\gamma_{0} r\right)-c^{2} \frac{\partial}{\partial r}\left(\frac{a_{1}}{r}\right)+\frac{c \beta_{1}}{r}$

$$
=-2 \kappa^{2} \gamma_{0} r c
$$

subject to $a_{1}=0, \partial \beta_{1} / \partial r=0, \frac{\partial}{\partial r}\left(\gamma_{1} r\right)=0$, when $r=r_{0}$. Eliminating $a_{1}$ and $\gamma_{1}$, the equation to determine $\beta_{1}$ is

$$
\frac{\partial}{\partial r}\left(r \frac{\partial \beta_{1}}{\partial r}\right)-\frac{\beta_{1}}{r}=-\frac{2}{c^{2}} \gamma_{0} r
$$

all but the most important terms being neglected; hence

$$
\beta_{1}=B r-\frac{A r}{c^{2}} \log r
$$


and making use of the boundary condition this becomes

$$
\beta_{1}=\frac{A r}{c^{2}}\left(1-\log \frac{r}{r_{0}}\right)
$$

Substituting this value of $\beta_{1}$ in the above equations, and rejecting all but the most important terms, they become

$$
a_{1}=\frac{\partial}{\partial r}\left(\gamma_{1} r\right), \quad \frac{\partial}{\partial r} r \frac{\partial}{\partial r}\left(\gamma_{1} r\right)-\gamma_{1}=\frac{A}{c} ;
$$

whence

$$
\gamma_{1}=C+\frac{A}{2 c} \log r
$$

and therefore, determining $C$ from the boundary condition,

$$
\gamma_{1}=\frac{A}{2 c}\left[\log \frac{r}{r_{0}}-1\right], \quad \alpha_{1}=\frac{A}{2 c} \log \frac{r}{r_{0}} .
$$

The function $\gamma_{0}$ has now to be determined to the same order as the order of magnitude of $\alpha_{1}, \beta_{1}, \gamma_{1}$; assuming that a quantity multiplied by $\kappa^{2} c^{2}-1$ is more important than the same quantity multiplied by $(r / c)^{2}$ for the values of $r$ considered, the equation to determine $\gamma_{0}^{\prime}$ where $\gamma_{0}=A / r+\gamma_{0}^{\prime}$ is

whence, writing

$$
\frac{\partial}{\partial r} \frac{1}{r} \frac{\partial}{\partial r}\left(\gamma_{0}^{\prime} r\right)+\left(\kappa^{2}-\frac{1}{c^{2}}\right) \frac{A}{r}=0
$$

$$
\kappa^{2}-\frac{1}{c^{2}}=\frac{\lambda^{2}}{c^{2}}, \quad \gamma_{0}^{\prime}=D r-\frac{\lambda^{2} A}{2 c^{2}} r \log r,
$$

and, determining $D$ from the boundary condition,

$$
\gamma_{0}=\frac{A}{r}+\frac{A \lambda^{2} r}{2 c^{2}}\left[\frac{1}{2}-\log \frac{r}{r_{0}}\right] .
$$

The components of the magnetic force in the immediate neighbourhood of the wire are therefore approximately given by the real parts of

$$
\begin{aligned}
\alpha & =\frac{A}{2 c} \log \frac{r}{r_{0}} \cos \theta \sin \phi, \\
\beta & =\frac{A r}{c^{2}}\left(1-\log \frac{r}{r_{0}}\right) \sin \theta \sin \phi, \\
\gamma & =\frac{A}{r} \cos \theta+\frac{A \lambda^{2} R}{2 c^{2}}\left[\frac{1}{2}-\log \frac{r}{r_{0}}\right] \cos \theta+\frac{A}{2 c}\left[\log \frac{r}{r_{0}}-1\right] \cos \theta \cos \phi,
\end{aligned}
$$

and the components of the electric force by the real parts of

$$
X=\frac{\iota V A}{\kappa c r} \sin \theta, \quad Y=-\frac{\iota V \lambda^{2} A}{\kappa c^{2}} \log \frac{r}{r_{0}} \cos \theta, \quad Z=\frac{\iota V A}{2 \kappa c^{2}} \log \frac{r}{r_{0}} \sin \theta \sin \phi,
$$


all but the most important terms in these latter expressions being neglected. The corresponding expressions for the oscillations of shorter wave-lengths can be obtained in the same manner. It follows that for a thin wire whose central axis forms a circle the electric and magnetic forces in the immediate neighbourhood of the wire are not the same as those due to a circuit of Hertzian oscillators however thin the wire may be, but the component electric force normal to the wire at its surface is very approximately the sume as that due to a circuit of Hertzian oscillators arranged so that the line integral of the magnetic force looping the circuit close to it is the real part of $2 \pi A \cos \theta$. It can be inferred that whatever the form of the circuit formed by the central axis of $a$ closed thin wire may be, provided it is of continuous curvature, the electric force normal to the wire at its surface is for the oscillations of greatest wavelength very approximately the same as that due to a circuit of Hertzian oscillators arranged so that the line integral of the magnetic force looping the circuit close to it is the real part of $2 \pi A \cos 2 \pi s / s_{0}$, where $s_{0}$ is the length of the circuit.

It remains to determine the electric and magnetic forces at a distance from the wire. Let $O$ be a point $x, y, z$ at a distance from the wire; then the component parallel to the axis of $x$ of the electric force at $O$ is given by

$$
X=\frac{\imath}{4 \pi \kappa} \iint M\left[l_{1} \frac{\partial^{2}}{\partial x^{2}}+m_{1} \frac{\partial^{2}}{\partial x \partial y}+n_{1} \frac{\partial^{2}}{\partial x \partial z}+\kappa^{2} l_{1}\right] \frac{e^{-\iota \kappa l l}}{R} d S,
$$

where $l_{1} M, m_{1} M, n_{1} M$ are the components of the electric flow on the surface of the wire at the point $Q\left(x^{\prime}, y^{\prime}, z^{\prime}\right)$ on it and

$$
R^{2}=\left(x-x^{\prime}\right)^{2}+\left(y-y^{\prime}\right)^{2}+\left(z-z^{\prime}\right)^{2} .
$$

If $P$ is the point $x_{1}, y_{1}, z_{1}$ on the central axis of the wire, $P Q$ being perpendicular to the central axis, $l_{2}, m_{2}, n_{2}$ the direction cosines of the tangent to the central axis at $P$, and $l_{3}, m_{3}, n_{3}$ the direction cosines of the tangent to the wire at $Q$ perpendicular to $P Q$, then, in the previous notation, $l_{1} M=l_{2} \gamma+l_{3} \beta, \ldots$, and $R^{2}=R_{1}^{2}+r_{0}^{2}+2 R_{1} r_{0} \sin \chi \cos \left(\phi-\phi^{\prime}\right)$, where $R_{1}$ is the distance $O P, r_{0}$ is the radius of the wire, $\chi$ is the angle $O P$ makes with the tangent to the central axis at $P$, and $\phi-\phi^{\prime}$ is the angle between the two planes drawn through the tangent at $P$ and the points $O$ and $Q$. The integrals to be evaluated are all of the form

$$
\iint\left(l_{2} \gamma+l_{3} \beta\right) \frac{e^{-\imath \kappa l}}{R} d S,
$$


that is

$$
\begin{aligned}
\iint\left(l_{2} \gamma+l_{3} \beta\right) R_{1}^{-\frac{1}{2}} r_{0}^{-\frac{b}{2}} e^{\frac{1}{3}(n c)} \sum_{0}^{\infty}(2 n+1) e^{\frac{z}{3}(n \pi)} K_{n+\frac{1}{3}}\left(\iota \kappa R_{1}\right) J_{n+\frac{1}{3}}\left(\kappa r_{0}\right) \\
\times P_{n}\left\{\sin \chi \cos \left(\phi-\phi^{\prime}\right)\right\} r_{0} d \phi d s,
\end{aligned}
$$

$R_{1}$ being greater than $r_{0}$. Now the order of the ratio of $K_{n+\frac{1}{2}}\left(i \kappa R_{1}\right) J_{n+\frac{1}{3}}\left(\kappa r_{0}\right)$ to $K_{\frac{1}{2}}\left(\kappa R_{1}\right) J_{\frac{1}{2}}\left(\kappa r_{0}\right)$ is that of $\left(\kappa r_{0}\right)^{n}$ or $\left(r_{0} / R_{1}\right)^{n}$, whichever is the greater of these two quantities; and, if the shortest distance of the point $O$ from the central axis of the wire is greater than $\lambda / 2 \pi$, where $\lambda$ is the wave-length of the oscillations, the order is that of $\left(\kappa r_{0}\right)^{n}$. When the central axis is a circle of radius $c, \beta$ is at most of the order $r_{0} / c$; hence, if $r_{0}$ is so small that $\left(\kappa r_{0}\right)^{2}$ can be neglected, the value of the above integral is

$$
2 \pi \int l_{2} \gamma_{0} \frac{e^{-\iota \kappa l_{1}}}{R_{1}} d S
$$

and the component parallel to the axis of $x$ is the real part of

$$
-\frac{\iota V}{2 \kappa} \int \gamma_{0}\left(l_{2} \frac{\partial^{2}}{\partial x^{2}}+m_{2} \frac{\partial^{2}}{\partial x \partial y}+n_{2} \frac{\partial}{\partial x \partial z}+\kappa^{2} l_{2}\right) \frac{e^{\iota \kappa} R_{1}}{R_{1}} d s .
$$

Therefore at a point whose shortest distance from the central axis of the wire is of the same order of magnitude as $\lambda / 2 \pi$ the electric and magnetic forces are sensibly the same as those due to a circuit of Hertzian oscillators arranged along the central axis of the wire, provided the radius $r_{0}$ of the wire is so small that $\left(\kappa r_{0}\right)^{2}$ can be neglected. It will be observed that the arrangement of Hertzian oscillators is the same as that which produces sensibly the same electric force normal to the wire at its surface. As before, it can be inferred that this result holds for a thin wire, whatever the form of the closed circuit made by it may be, provided the curvature of the circuit is continuous.

If a tubular surface be described by a sphere of radius $R_{1}$ of the same order as $\lambda / 2 \pi$, the centre of the sphere moving along the central axis of the wire, the amount of energy that crosses this surface in a half period is less than the amount of energy $E$ radiated out in a half period from the circuit of Hertzian oscillators which arranged along the central axis produce sensibly the same electric and magnetic forces at points outside this surface, the amplitudes being supposed kept constant during this half period. Now $E$ is a finite quantity depending on the line integral of the magnetic force at the surface of the wire looping the circuit, and, if this be kept constant while $r_{0}$ is diminished, $E$ will remain sensibly constant, but the amount of energy inside the tubular surfaces increases indefinitely as $r_{0}$ is diminished ; therefore the rate of decay of the oscillations can be 
made less than any assigned small quantity, however small, by sufficiently diminishing the radius of the wire, but it cannot be made to vanish; for, as has been seen, the limit of a conducting wire when the radius is diminished without limit has no existence.

It is perhaps unnecessary to add that this result ceases to hold when the circuit is not closed; for then the electric force in the immediate neighbourhood of a free end is indefinitely great compared with the electric force at the surface of the wire at a distance from a free end. For example, in the case of a resonator, supposed to be a thin wire with its free ends $A$ and $B$ close to each other, the electric forces at $A$ and $B$ at any instant of time are of opposite sign and very great: thus there are a very great number of lines of electric force going from the immediate neighbourhood of $A$ to the immediate neighbourhood of $B$. The number of lines of force that close up in the neighbourhood of the gap each half period is therefore indefinitely great compared with the number that can close up near any node on the surface of the wire, and therefore the radiation from the gap is indefinitely great compared with that due to the remainder of the surface of the wire, and, although the latter can be neglected, the former cannot. 